\title{
Learning from the EPOCH trial (Editorial) \\ What we have learnt from a trial of an intervention to improve survival following emergency laparotomy?
}

\section{Tim Stephens ${ }^{1}$ and Rupert M. Pearse ${ }^{1}$}

1. Barts \& The London School of Medicine and Dentistry, Queen Mary University of London, UK

Correspondence to:

Tim Stephens

Critical Care and Perioperative Medicine Research Group,

William Harvey Research Institute, Queen Mary University of London

c/o ACCU Research Team

Royal London Hospital

London

E1 1BB

United Kingdom

e-mail: t.t.stephens@qmul.ac.uk

Tel: +442035940351

Main text: 1082 words

Keywords: Quality improvement; Postoperative care/methods; Surgical Procedures,

Operative/mortality; 


\section{Introduction}

In a recent Lancet publication, we reported the findings of a stepped-wedge, cluster randomised trial of a quality improvement intervention to enhance the outcomes of emergency abdominal surgery [1]. In this editorial we summarise the possible factors that led to the failure of the intervention to impact on patient outcomes and what has been learnt from the trial.

The Enhanced Peri-Operative Care for High-risk patients (EPOCH) trial had two interventions: a 37component care-pathway, representing best care for this patient group and a quality improvement programme designed to support implementation of the pathway in 93 hospitals across the UK. National Health Service (NHS) hospitals contributing to the National Emergency Laparotomy Audit were eligible for inclusion [2]. Local improvement leads from each perioperative discipline (surgery, anaesthesia, and critical care) were responsible for leading hospital wide improvement efforts to implement the care pathway. The quality improvement programme was designed to support these local clinicians and comprised of educational meetings, a virtual learning environment and telephone / email support from the national EPOCH team. The programme was designed to be typical of NHS improvement efforts at the time and had minimal face-to-face contact time, recognising the limited resources of the study and of clinician time within the NHS. Full details about the EPOCH improvement programme are reported elsewhere [3]. The intervention ran for 80 weeks in total (the first cluster began the intervention 5 weeks into the study), with one cluster commencing the intervention every 5-week step from the second to the 16th time period.

The primary objective of the EPOCH trial was to reduce 90 -day mortality from $25 \%$ to $16 \%$. Both the intervention and usual care groups had a 90 -day mortality of $16 \%$. The most frequently enrolled patients had intestinal obstruction or perforation. Process measures indicate that the intervention group were more likely to have preoperative documentation of risk, to receive goal directed fluid therapy and to have serum lactate measured at the end of surgery than the usual care group. Other key process improved less, such as patients entering the operating theatre within their target timeframe, critical care admission post-operatively and consultant decision to operate. Secondary outcomes, including 180-day mortality, length of stay, and readmissions, also did not differ between the two groups. 


\section{Why didn't the EPOCH interventions work?}

The trial process evaluation revealed good engagement with the quality improvement programme amongst clinicians but limited time and resources for staff to implement change. There were wide variations in intervention fidelity between hospitals, with differences in the both the components within the care-pathway that teams tried to implement first, and the quality improvement approaches teams used to make change happen. This created wide variations in the rate of change and eventual success across the 93 hospitals. The trial hypothesis was based on the belief that limited awareness of poor outcomes was the primary barrier to better patient care. In reality, the reasons for poor quality care were much more complex. As a result, local EPOCH teams were asked to tackle too many parts of the patient care pathway, with insufficient quality improvement support and training, and perhaps most importantly, in too short a timeframe. Whilst patient survival did not improve, EPOCH provided vital learning about how we can and can't improve patient care using large-scale quality improvement programmes.

There is some debate regarding the value of large trials which frequently fail to confirm the clinical effectiveness of interventions. There is too much emphasis on trials with 'positive' findings, prioritising publication of trials of interventions that 'work' whilst failing to appreciate the wealth of clinical information provided by a well-conducted clinical trial, whether 'positive' or 'negative'. This reflects frustration in the clinical community when a treatment or intervention simply does not deliver on the initial promise when it is rigorously evaluated. So, what are the lessons from a 'negative trial' like EPOCH? Firstly, we do not doubt the value of the core clinical components of the intervention but rather the methods for improving care at the frontline. Improvement science has developed substantially since the EPOCH trial was designed back in 2012-13 and improvement methods, such as audit and feedback and the Plan-Do-Study-Act cycle, may well be effective. They are not, however, quick and easy fixes to a problem, and require adequate manpower, a realistic timeframe and enough resource to make them work. Also, some system-level improvements, such as reducing the time taken to get the patient to surgery, may be beyond the scope of what frontline clinicians can change by themselves. Quality improvement projects cannot make up for major structural issues such as too few emergency operating theatres. Without addressing these issues, quality improvement efforts led by frontline colleagues will continue to fail. Next, by doing EPOCH we have confirmed what is necessary to trial a complex intervention such as a quality improvement programme; not only does the trial design need to account for temporal trends toward improvement (the so called secular trend) but also requires careful consideration of which process measures to collect and to integrate a mixed methods 
process evaluation, to detail what happened 'on the ground' during the trial. If we had run the EPOCH trial without these additional research elements it would have been a genuinely negative result, as we would not have understood why we got the result we did.

\section{Improving emergency surgical care}

The volume and acuity of emergency general surgery will continue to increase with our aging, multimorbidity population [4]. Whilst the EPOCH trial did not result in improved outcomes, it must not deter future efforts to improve care. Indeed, by clearly outlining the challenges involved in reducing variation and implementing best practices, for patients requiring emergency general surgery, we hope to make future quality improvement endeavours more effective. 


\section{Declaration of interests}

RP holds research grants and has given lectures and/or performed consultancy work for BBraun, Glaxo Smithkline, Intersurgical, and Edwards Lifesciences, and is a member of the Associate editorial boards of the British Journal of Anaesthesia, British Journal of Surgery and BMJ Quality and Safety.

\section{Funding}

$\mathrm{RP}$ is a National Institute for Health Research Professor.

\section{Authors' contributions}

Both authors were responsible for drafting the manuscript and approved the submitted version. 


\section{References}

[1] Peden CJ, Stephens TJ, Martin G, Kahan B, Thomson A, Rivett K, et al. Effectiveness of a national quality improvement programme to improve survival after emergency abdominal surgery: A stepped-wedge cluster randomised trial. Lancet 2019;393.

[2] The National Emergency Laparotomy Project Team. The first patient report of the national emergency laparotomy audit. 2015.

[3] Stephens TJ, Peden CJ, Pearse RM, Shaw SE, Abbott TEF, Jones EL, et al. Improving care at scale: process evaluation of a multi-component quality improvement intervention to reduce mortality after emergency abdominal surgery (EPOCH trial). Implement Sci 2018;13:142. doi:10.1186/s13012-018-0823-9.

[4] Fowler AJ, Abbott T, Prowle J, Pearse RM. The age of patients undergoing surgical procedures in England: An ecological study using Hospital Episode Statistics. Br J Surg 2019. 